\title{
The crosstalk between the gut microbiota and lipids ${ }^{\text {is }}$
}

\author{
Philippe Gérard* \\ Micalis Institute, INRAE, AgroParisTech, Université Paris-Saclay, 78350 Jouy-en-Josas, France
}

Received 4 September 2020 - Accepted 18 November 2020

\begin{abstract}
The human intestine harbours a complex and diverse bacterial community called the gut microbiota. This microbiota, stable during the lifetime, is specific of each individual despite the existence of a phylogenetic core shared by the majority of adults. The influence of the gut microbiota on host's physiology has been largely studied using germfree animals and studies using these animal models have revealed that the effects of lipids on host physiology are microbiota-dependent. Studies in mice have also shown that a high-fat diet rapidly and reproducibly alters the gut microbiome. In humans, dietary fat interventions did not lead to strong and consistent modifications of the microbiota composition. Nevertheless, an association between total fat intake and the reduction of the microbiota richness has been repeatedly found. Interestingly, different types of fat exert different or even opposite effects on the microbiota. Concurrently, the gut microbiota is able to convert the lipids entering the colon, including fatty acids or cholesterol, leading to the production of metabolites with potential health effects.
\end{abstract}

Keywords: Microbiome / high-fat diet / germ-free mice / cholesterol / fatty acids

Résumé - Les relations bidirectionnelles entre le microbiote intestinal et les lipides. L'intestin humain héberge une communauté bactérienne complexe et diverse appelée microbiote intestinal. Ce microbiote, stable au cours de la vie, est spécifique à chaque individu malgré l'existence d'un noyau phylogénétique partagé par la majorité des adultes. L'influence du microbiote intestinal sur la physiologie de l'hôte a été largement étudiée en utilisant des animaux sans germes. Ces études ont en particulier révélé que les effets des lipides sur la physiologie de l'hôte sont dépendants du microbiote. Chez l'animal il a également été montré qu'un régime alimentaire riche en lipides modifie rapidement et de manière reproductible le microbiote intestinal. Chez l'homme, des études nutritionnelles impliquant des lipides n'ont pas entrainé de modifications fortes et cohérentes de la composition du microbiote. Néanmoins, une association entre la consommation de lipides et la réduction de la richesse du microbiote a été constatée à plusieurs reprises. Il est intéressant de noter que différents types de lipides exercent des effets distincts, voire opposés, sur le microbiote. Par ailleurs, le microbiote intestinal est capable de convertir les lipides présents dans le côlon, en particulier les acides gras ou le cholestérol, aboutissant à la production de métabolites ayant des effets potentiels sur la santé.

Mots clés : Microbiome / régime hyperlipidique / souris sans germes / cholestérol / acides gras

\section{Introduction}

The human digestive tract is home to more than 100000 billion microorganisms, mainly bacteria and archaea, which make up the intestinal microbiota. The microorganisms that colonize us are responsible for many functions essential to maintaining our health, to the point that we can consider this microbiota as an additional organ of our body. The dialogue that is established between this microbiota and our gut can also be modulated by various factors, in particular food. In this review, we will be particularly interested in the bidirectional relationships between the gut microbiota and lipids.

\section{The gut microbiota}

Although data exist regarding gut colonization before birth, it is widely accepted that humans' first exposure to microbes occurs mainly in the birth canal. At birth, newborn babies experience rapid colonization by microbes from their mothers and the surrounding environment. The composition of

\footnotetext{
Contribution to the Topical Issue "Microbiota, nutrition and lipids: consequences on health / Microbiote, nutrition et lipides : conséquences sur la santé".

*Correspondence: philippe.gerard@inrae.fr
} 
this infant microbiota depends on various factors including the mode of birth (cesarean section or vaginal delivery), antibiotic treatment, diet (breastfeeding or infant formula) or environmental sanitation (Palmer et al., 2007). Fluctuating at the start of life, the assembly of the human intestinal microbiota becomes balanced after a few years in children and then remains relatively stable over time in healthy adults, slightly fluctuating around an individual nucleus of stable colonizers, in the absence of external disturbances (infections, antibiotics...). Then, alterations occur in old age together with digestive physiology and diet changes (Faith et al., 2013). In adults, diet has been demonstrated to be the factor having the highest influence on gut microbiota composition. Other factors, including genetics, early environmental exposures, or lifestyle, impact gut microbiota leading to a composition which is unique and specific of each individual.

The majority of the gut bacteria still remains impossible to cultivate, so our understanding of this microbiota has long been limited by technical problems. The development of molecular tools and sequencing techniques now makes possible a reliable evaluation of the intestinal microbiota, showing that each gut microbiota is made up of 500 to 1000 bacterial species. Three bacterial phyla, Firmicutes, Bacteroidetes and Actinobacteria, account for the majority of the dominant intestinal bacteria. In addition, species belonging to the Archae domain are present in the dominant fecal microbiota of around half of adults in Western countries, while the presence of fungi is also now well documented (Knight et al., 2017). The increase in sequencing and bioinformatics capacities has made it possible to not only access to the type of bacterial species present but also to their genetic content, showing that each individual harbors an average of 500 to 600000 bacterial genes, of which about half are shared by most individuals (Li et al., 2014). However, many functions are carried by the gut microbiota in only a fraction of humans and could contribute greatly to the metabolic diversity observed in the human population. Among the main functions of the microbiota, the fermentation of substrates available in the colon, the metabolism of food toxins and carcinogens, the role of barrier to colonization by pathogenic microorganisms, the synthesis of vitamins, the development and maturation of the intestinal immune system and interactions with epithelial cells help maintain the health of the host (Gérard and Bernalier-Donadille, 2007). In addition, imbalances in its composition (i.e. dysbiosis) have been associated with immune disorders, susceptibility to infections, and more recently with several non-intestinal pathologies including cardiovascular disease, obesity, diabetes, liver or even brain disease (Duca et al., 2014; Gérard, 2016; Lloyd-Price et al., 2016; Safari and Gérard, 2019).

\section{The gut microbiota influences the effects of lipids on host physiology}

Germ-free (GF) animal models have been used for decades to define the consequences of the absence of gut microbiota and therefore to establish the host physiological functions which are influenced by these bacteria. Using these animal models, it has been evidenced that the effects of dietary lipids on host physiology and metabolism are microbiota dependent.
First, in contrast to conventional mice, GF mice fed a high-fat diet (HFD) were found protected from diet-induced obesity (DIO) (Backhed et al., 2007). This lean phenotype was associated with increased skeletal muscle and liver levels of phosphorylated AMP-activated protein kinase (AMPK). Hence, the gut microbiota may inhibit skeletal muscle fatty acid (FA) oxidation through a metabolic pathway involving phosphorylation of AMPK. Moreover, the gut microbiota suppresses the expression of Angiopoietin-like 4, a circulating inhibitor of lipoprotein lipase. Therefore, deposition of triglycerides in adipocytes are decreased in GF mice contributing to the lean phenotype. It was further observed that GF mice receiving a HFD showed enhanced insulin sensitivity with improved glucose tolerance and reduced insulinemia in comparison to conventional mice. This was associated with a reduced hypercholesterolemia, a moderate accretion of hepatic cholesterol and an increase in fecal cholesterol excretion suggesting an altered cholesterol metabolism in GF mice (Rabot et al., 2010). However, the absence of gut microbiota does not provide a general protection from DIO which may depend on mice genetic background and diet composition. Indeed, C3H GF mice were not found resistant to the obesogenic effect of a low-sucrose, lard-based HFD, while resistant to high sucrose, palm oilbased HFD (Fleissner et al., 2010). Moreover, GF mice, but not conventional mice, were DIO resistant when fed a cholesterolrich lard based HFD, whereas on a cholesterol-free palm oilbased HFD, DIO was independent of the gut microbiota (Kübeck et al., 2016). This indicates that the gut microbiota may be responsible for the distinct effects of different fat types on host metabolism and it was proposed that an interaction of gut microbiota and dietary cholesterol is essential for fat accretion in mice. This was confirmed in a study where Conventional and GF mice were fed palm oil or lard diet supplemented with bile acids. In combination with bile acids, dietary lard enhanced fat mass accumulation in colonized, but not in GF mice when compared to palm oil. This was associated with impaired glucose tolerance, lower fasting insulin levels, lower counts of enteroendocrine cells, and increased steatosis (Just et al., 2018). This indicates that lard in the diet had a detrimental impact on host metabolism when combined with bile acids, but only in the presence of endogenous gut microbes. This also rises the question whether gut microbiota composition may influence the effects of lipids on host physiology. Indeed, it has been demonstrated that housing conditions and associated changes in gut bacterial colonization are pivotal for maintenance of gut barrier integrity in DIO mice (Müller et al., 2016). Also, HFD-induced glucose intolerance has been shown to depend on the composition of the gut microbiota while obesity did not (Rabot et al., 2016). These studies highlight the need for taking into account both the dietary fat source and microbiota composition when interpreting data from diet-induced obesity models.

\section{Dietary and endogenous lipids in the intestine}

The lipids contained in colonic contents derived from the undigested residue of dietary fat, from endogenous secretions and desquamation of colonocytes, as well as from bacterial origins. In general, only a small proportion of the dietary fat 
consumed by humans reaches the large intestine despite huge inter-individual variations in lipids absorption in the small intestine. Yet, diet rich in lipids, certain clinical conditions, and drugs that inhibit pancreatic lipases or adsorbents lead to increased lipid flow to the large intestine. It has been estimated that an average of $100 \mathrm{~g}$ of triacylglycerols (TAGs) and 4 to $8 \mathrm{~g}$ of phospholipids are consumed daily in western adult population, of which more than $75 \%$ are absorbed in the small intestine. The amount of total lipids that reach the colon under physiological conditions has been evaluated at between 5 and $8 \mathrm{~g}$ per day in humans. Interestingly, it was recently shown that the small intestinal microbiota is necessary for efficient digestion and absorption of lipids in mice (Martinez-Guryn et al., 2018), while its role in the digestion and absorption of lipids in humans is currently unknown. Moreover, the nature of the lipid entering the small intestine and its absorption through the stomach wall directly affects the amount and type of lipid entering the large intestine. Indeed, dietary medium-chain FA are completely absorbed by the upper gastrointestinal tract while unsaturated FA are much more easily absorbed than saturated FA. Much higher gastrointestinal lipid loads are observed in people with certain clinical conditions, including those with cystic fibrosis or pancreatic deficiency, or those who have undergone GI resection (Juste, 2005). Similarly, anti-obesity drugs that work by inhibiting gastrointestinal lipases, decrease fat absorption leading to increased lipid load in the colon. The host itself and its commensal bacteria contribute to the fats in the digestive tract. Host-derived lipids are derived from bile, intestinal secretions and desquamation of the intestinal epithelium, and are rich in cholesterol and phospholipids (Gérard, 2020). Phospholipids, sphingolipids and a large variety of saturated and unsaturated FA from the dead cells of gut bacteria also contribute to the pool of lipids in the colon and it has been estimated that between one-quarter and one-third of all lipids excreted in human feces are of bacterial origin.

All these lipids reaching the colon can both modified gut microbiota composition and activity of the gut microbiota. They can also be modified by the gut bacteria leading to changes in their health effect. This interrelationship between lipids and the gut microbiota will be developed in the following parts of the review.

\section{Effects of lipids on the gut microbiota}

\subsection{Evidences from rodent studies}

Initial evidences that the dietary fat intake impacts gut microbiota composition originated from rodent studies whose microbiota composition resembles the human microbiota at the phylum and family levels. Indeed, HFD are commonly used to trigger obesity and metabolic diseases in rodents and this has been largely associated with gut microbiota alterations (Busnelli et al., 2020; Safari et al., 2020). However, the heterogeneity of the studies regarding the quantity and the nature of fat in so called HFD constitutes one challenge to draw general conclusions. A recent meta-analysis examined the effect of HFD on the gut microbiota and revealed few reproducible gut microbiome alterations including a slight reduction in the bacterial diversity while the ratio of Firmicutes to Bacteroidetes was significantly increased in 15 out of the
25 studies included (Bisanz et al., 2019). An increase in species belonging to the Lachnospiraceae, Ruminococcaceae, and S24-7 Muribaculaceae families was also identified as a common effect of HFD. Interestingly, it was found that many of the structural alterations of the microbiome due to HFD are reversible and that restoration of a close to initial microbiota can be achieved in one week after stopping high-fat feeding in mice (Safari et al., 2019). The mechanisms by which dietary lipids affect gut microbiota are not well understood. Nonetheless, FA have a broad spectrum of antibacterial activities including inhibition of ATP production as well as lysis and solubilization of bacterial cell membranes. Interestingly, these bactericidal activities depend on the carbon chain length, saturation and double bond position of the FA (Zheng et al., 2005). Mice have also been used to assess the effect of different types of fat on the gut microbiota. As an example, isocaloric HFD containing either milk fat, corn oil or olive oil were shown to differently alter microbiota composition with olive oil being most distinct from the corn oil and milk fat (Abulizi et al., 2019). Similarly, mice fed a HFD containing lard, rich in saturated fat, were compared to mice fed an isocaloric HFD containing fish oil, rich in n-3 polyunsaturated fatty acids (PUFA). The lard rich diet promoted growth of Bacteroides, Turicibacter and Bilophila while the fish oil rich diet increased abundance of the beneficial Bifidobacteria, Akkermansia and Lactobacillus spp. (Caesar et al., 2015). However, fish oil may not always be beneficial as it was also shown that mice fed with fish oil had an higher relative abundance of phylum Proteobacteria and genus Desulfovibrio which have been associated with inflammation (Li et al., 2017). It has also been shown that a diet rich in milk fat, but not diets with lard or safflower oil, increased expansion of Bilophila wadsworthia which was proved to aggravate colitis as well as HFD induced metabolic dysfunctions (Devkota et al., 2012). This involves the promotion of taurine-conjugation of bile acids by milk fat, which increases the availability of sulfur used by $B$. wadsworthia. Recently, the effects of plant-based fat on gut microbiota modulation have been reviewed (Muralidharan et al., 2019). Compared to animal based fats, they favor a healthy gut microbiota including increases of Bifidobacteium spp., Lactobacillus spp., short-chain fatty acids (SCFA) producing bacteria, and decrease of Clostridum perfringens, Ruminococcaceae family, and Enterococcus. As an example, olive oil was shown to modify the gut microbiome differently than butter, with decreased Desulfovibrio and increased Lactobacillus and Clostridium cluster XIV, which were correlated with lower plasma insulin and lower systolic blood pressure, respectively. Finally, several studies have reported that HFD increases lipolysaccharides (LPS) expressing bacteria, like Enterobacteriaceae, leading to an elevated level of LPS in the circulation promoting a pro-inflammatory state called metabolic endotoxemia (Cani et al., 2007).

\subsection{Effects of lipids on the human gut microbiota}

Determining the impact of dietary fats on the gut microbiota in humans is more complicated than in rodents due to interindividual variation in the composition of the microbiome which may outweigh the effect of dietary 
intervention. Moreover, in order to maintain an isocaloric diet, the increase in fat quantity is made at the expense of other nutrients, usually carbohydrates so that it may be difficult to conclude that the microbiota effects are exclusively due to change in fat content. Nonetheless, it was shown that HFD rapidly and reproducibly alters the human gut microbiome with increased abundance of bile-tolerant bacteria including Alistipes, Bilophila, and Bacteroides, attributed to the high fat provision, and reduced abundance levels of Roseburia, Eubacterium, and Ruminococcus, due to the lack of fermentable carbohydrates (David et al., 2014). Recently, two reviews examined the results obtained in human observational and intervention studies (Wolters et al., 2019; Mokkala et al., 2020). Their main conclusions revealed association between total fat intake and the reduction of the microbiota richness and diversity while PUFA had no effect on these parameters. Regarding microbiota composition, dietary fat interventions did not lead to strong and consistent modifications. In observational studies, high intake of total fat or saturated FA correlated with the abundance of Clostridium boltae and Blautia respectively, which are both associated with unhealthy metabolic outcome. Conversely, a diet rich in PUFA correlated with increased populations of Tenericutes. Interestingly, omega-3 versus omega- 6 fats seem to have different or even opposite effects on microbiota composition. This has been highlighted in two recent randomized controlled trials in healthy adults: a daily intake of $4 \mathrm{~g}$ of the omega-3 eicosapentaenoic and docosahexaenoic PUFA for eight weeks did not lead to diversity or phyla composition changes. However, a reversible increased abundance of several genera, including Bifidobacterium, Roseburia and Lactobacillus was observed during the intervention (Watson et al., 2018). More recently, a 6-month trial analyzed the effects of three isocaloric diets differing in fat content, with $20 \%, 30 \%$, and $40 \%$ of energy from fat, respectively (Wan et al., 2019). Notably, the fat originated from soybean oil, rich in omega-6 PUFA. The lower-fat diet was associated with increased bacterial diversity, whereas the higher-fat diet was associated with increased Alistipes and Bacteroides and decreased butyrate producers including Faecalibacterium and Blautia. Accordingly, the concentrations of butyrate and total SCFA were significantly decreased in the higher-fat diet group in comparison with the other groups. Interestingly, the microbial metabolites p-cresol and indole, known to be associated with host metabolic disorders, increased together with the fat content. In addition, the higher-fat diet was associated with enrichment in the LPS biosynthesis pathway as well as elevated plasma proinflammatory factors after the intervention suggesting that microbiota changes may contribute to elevated inflammation associated with HFD consumption.

The very low carbohydrate high-fat ketogenic diet (KD) is a dietary protocol driving the body into ketosis and reliance on fat for energy. It has been used since the 1920 as a treatment for refractory epilepsy and it is currently getting popularity as a potential therapy for obesity and related metabolic disorders. Only few studies have looked at the impact of KD on the human gut microbiome. In a study in children for the treatment of intractable epilepsy, Zhang et al. detected a lower diversity after KD therapy and revealed significantly decreased abundance of Firmicutes and increased levels of Bacteroidetes (Zhang et al., 2018). Interestingly, they found a distinct microbiota composition in the non-responsive group (no reduction in seizures) suggesting that the microbiota may contribute to KD efficacy. More recently, it has been shown that KDs alter the human and mouse gut microbiota in a manner distinct from HFD. They particularly observed a decrease of Bifidobacterium due to the host production of ketone bodies that specifically inhibited bifidobacterial growth. They also showed that Bifidobacterium levels decreased with increasing carbohydrate restriction, thus highlighting that carbohydrate restriction, rather than highfat intake, may be the main contributor to the KD's impact on the gut microbiome (Ang et al., 2020).

Together, these findings in mice and humans support a role of dietary fat in shaping gut microbiota composition and highlight the necessity of considering both fat quantity and quality in microbiome research.

\section{Metabolism of lipids by the gut microbiota}

As indicated previously, triglycerides are present in the colonic content together with the gut microbiota. The first step in the metabolism of triglycerides is their breakdown into free FA and glycerol by lipases. If a large variety of microorganisms possess lipase activity, very few are present in the human gut. Nevertheless, several intestinal bacteria from the Coriobacteriaceae family, including species belonging to the Collinsella and Eghertella genera were found lipase positive (Thorasin et al., 2015). Whether the lipase activity of these bacteria plays a role in host lipid metabolism in currently unknown. Intestinal bacteria also possess phospholipase $\mathrm{C}$ activity, which converts phospholipids into 1,2-sn-diacylglycerols (1,2-sn-DG) that can act as secondary messengers involved in cell growth and division. Interestingly, concentrations of 1,2-sn-DG in human feces are extremely variable among population suggesting a wide range of phospholipase activity in human gut microbiota. Active bacterial strains belonging to Firmicutes, Enterobacteria, and Actinobacteria phyla have been isolated indicating that phospholipase activity is present in phylogenetically distant species (Vulevic et al., 2004).

The colon also receives up to $1 \mathrm{~g}$ per day of cholesterol, $70 \%$ of which comes from bile, $20 \%$ of the fraction of food not absorbed in the small intestine and the remaining $10 \%$ of the desquamation of the intestinal mucous membranes (Bourgin et al., 2020). As early as the 1930s, it was shown that the intestinal microbiota was able to convert this cholesterol into coprostanol, not absorbed by the intestine and eliminated in the feces. This cholesterol metabolism follows a bimodal distribution within the human population, with a majority of high converters (almost complete cholesterol conversion) and a minority of low or inefficient converters (Kriaa et al., 2019). Two major pathways have been proposed for the conversion of cholesterol to coprostanol (Fig. 1). The first pathway involves direct reduction of the 5-6 double bond. The second pathway starts with the oxidation of the $3 \beta$-hydroxy group and isomerization of the double bond to yield 4-cholesten-3-one, which undergoes two reductions to form coprostanone and then coprostanol. This second pathway is supported by the presence of coprostanone in human feces and by the reduction of intermediate products to coprostanol by fecal samples. Nevertheless, both pathways may coexist in the human gut (Gérard, 2014). If this metabolism has been 

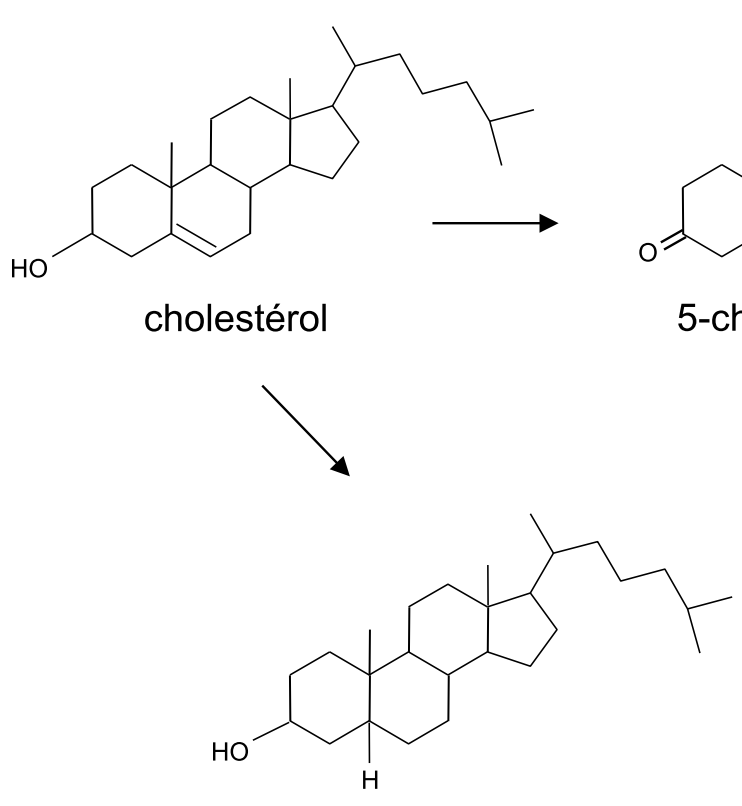

coprostanol

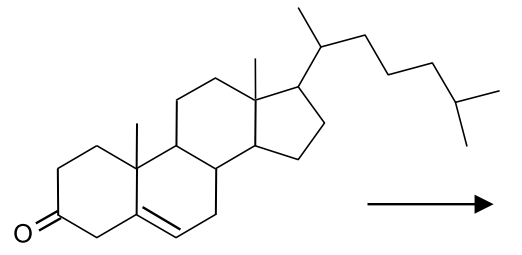

5-cholestèn-3-one<smiles>CC(C)CCCC(C)C1CCC2C3CCC4=CC(=O)CCC4(C)C3CCC12C</smiles>

4-cholestèn-3-one

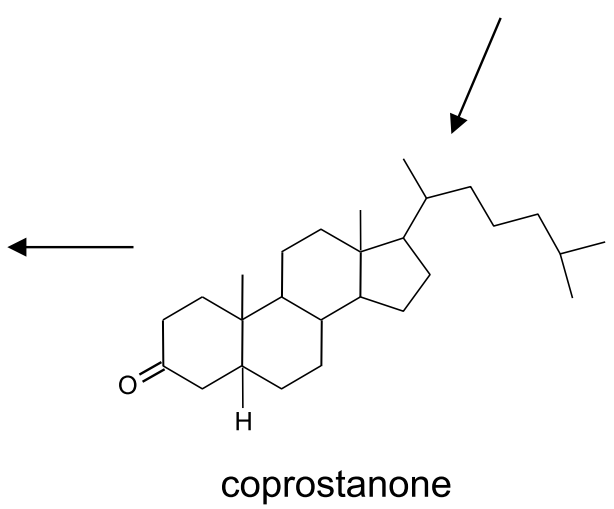

Fig. 1. Direct and indirect pathways for cholesterol-to-coprostanol conversion by the gut bacteria.

described for decades, the bacteria responsible remain largely unknown and only a few active strains have been first isolated from rat and baboon feces and from a hog sewage lagoon. The latter was named Eubacterium coprostanoligenes ATCC $51222^{\mathrm{T}}$ and it was shown to use an indirect pathway involving the formation of 4-cholesten-3-one to convert cholesterol to coprostanol. More recently, the first and still unique cholesterol-reducing bacterium from human origin has been isolated and characterized (Gérard et al., 2007). Unlike all other cholesterol-reducing strains isolated so far, this isolate named Bacteroides sp. strain D8 belongs to the Bacteroidetes phylum showing that distantly related bacteria may be responsible for this conversion. Very recently, Kenny et al. (2020) combined bioinformatic and biochemical approaches and identified for the first time cholesterol dehydrogenases from gut microbiomes that contribute to the metabolism of cholesterol to coprostanol. The cholesterol dehydrogenases are encoded by intestinal sterol metabolism A (ism A) genes in uncultured members of cluster IV Clostridium and were shown to be prevalent across geographically diverse human cohorts. The presence of bacteria with these genes correlated with lower levels of faecal and serum cholesterol, which suggest that conversion of cholesterol to coprostanol by the gut bacteria decreases host cholesterol levels in the intestine and serum (Kenny et al., 2020).

Intestinal bacteria can also react with FA, mainly through hydration and biohydrogenation of the unsaturated bonds in the aliphatic chain of monounsaturated fatty acids (MUFA) and PUFA. As an example, hydroxystearic acids formation from unsaturated FA is a widespread activity in the gut microbiota. Biohydrogenation has particularly been studied for the C18 unsaturated FA linoleic acid (LA) which is converted to rumenic acid which is hydrogenated to vaccenic acid (VA), and then to stearic acid. In addition, some human gut bacteria were found to perform a hydration step in which LA is converted to a hydroxy-C18:1 fatty acid (HFA), which was found to be a precursor of conjugated linoleic acid (CLA). In contrast to the rumen, human gut bacteria producing HFA, CLA and VA were found to belong to a wide variety of phylogenetic groups, including species of the genera Butyrivibrio, Lactococcus, Propionibacterium, Bifidobacterium, Faecalibacterium, Eubacterium, and Roseburia (Devillard et al., 2007). CLA can be also produced by several Lactobacillus species through a different pathway. Notably, CLA has been shown to modulate the immune system, and inhibit carcinogenesis, atherosclerosis and body fat in different animal models with distinct effects of the different CLA isomers. Interestingly, different bacteria produce different ratios of CLA isomers leading to different health effects depending on gut microbiota composition.

Finally, besides conversion of dietary lipids, the gut microbiota produces bioactive lipids that may pass the epithelial barrier and interact with host metabolism. One example is the recently identified sphingolipids produced by gut bacteria belonging to the Bacteroides genus that were shown to enhance the pool of sphingolipids available to the host and to modulate the level of bioactive lipids in the liver of mice (Johnson et al., 2020).

\section{Conclusion}

The interactions between the lipids and the gut microbiota are bidirectional. First, it is clear that dietary fats shape the gut microbiota composition with effects depending on the quantity and quality of the lipids. Also, the gut microbiota is able to metabolize the part of the lipids that enter the colon and to produce lipid metabolites that may impact host health. A poor explored issue is how this relationship influences host lipid metabolism. Comparison of GF and conventional mice has shown that the gut microbiota affects lipid composition in the serum, adipose tissue, and liver, with its greatest effect on triglyceride and phosphatidylcholine 
species (Velagapudi et al., 2010). Moreover, the gut microbiota induces hepatic production of MUFA and elongation of PUFA and regulates FA synthesis in the liver. The gut microbiota may also impact blood lipid levels and it was found in humans that triglycerides were higher and HDLcholesterol were lower in individual with low microbiota diversity (Le Chatelier et al., 2013). Moreover, it was recently revealed that the gut microbiota regulates host cholesterol homeostasis (Le Roy et al., 2019). Finally, a cross-validation analysis performed on a cohort study revealed that the gut microbiome contributes to a substantial proportion of the variation in blood lipids, with $6 \%$ of variance of serum triglycerides and $4 \%$ in HDL attributed to the microbiota composition (Fu et al., 2015). This highlights that the gut microbiota must be taken into account when one aims to assess the effects of lipids on health and disease.

\section{References}

Abulizi N, Quin C, Brown K, Chan YK, Gill SK, Gibson DL. 2019. Gut Mucosal Proteins and Bacteriome Are Shaped by the Saturation Index of Dietary Lipids. Nutrients 11: 418. https://doi. org/10.3390/nu11020418.

Ang QY, Alexander M, Newman JC, et al. 2020. Ketogenic Diets Alter the Gut Microbiome Resulting in Decreased Intestinal Th17 Cells. Cell 181: 1263-1275. https://doi.org/10.1016/j. cell.2020.04.027.

Backhed F, Manchester JK, Semenkovich CF, Gordon JI. 2007. Mechanisms underlying the resistance to diet-induced obesity in germ-free mice. Proc Natl Acad Sci USA 104: 979-84.

Bisanz JE, Upadhyay V, Turnbaugh JA, Ly K, Turnbaugh PJ. 2019. Meta-Analysis Reveals Reproducible Gut Microbiome Alterations in Response to a High-Fat Diet. Cell Host Microbe 26: 265272. https://doi.org/10.1016/j.chom.2019.06.013.

Bourgin M, Labarthe S, Kriaa A, et al. 2020. Exploring the Bacterial Impact on Cholesterol Cycle: A Numerical Study. Front Microbiol 11: 1121. https://doi.org/10.3389/fmicb.2020.01121.

Busnelli M, Manzini S, Jablaoui A, et al. 2020. Fat-Shaped Microbiota Affects Lipid Metabolism, Liver Steatosis, and Intestinal Homeostasis in Mice Fed a Low-Protein Diet. Mol Nutr Food Res 64: e1900835. https://doi.org/10.1002/ mnfr.201900835.

Cani PD, Amar J, Iglesias MA, et al. 2007. Metabolic endotoxemia initiates obesity and insulin resistance. Diabetes 56: 1761-72.

Caesar R, Tremaroli V, Kovatcheva-Datchary P, Cani PD, Bäckhed F. 2015. Crosstalk between Gut Microbiota and Dietary Lipids Aggravates WAT Inflammation through TLR Signaling. Cell Metab 22: 658-68. https://doi.org/10.1016/j.cmet.2015.07.026.

David LA, Maurice CF, Carmody RN, et al. 2014. Diet rapidly and reproducibly alters the human gut microbiome. Nature 505: 55963. https://doi.org/10.1038/nature12820.

Devillard E, McIntosh FM, Duncan SH, Wallace RJ. 2007. Metabolism of linoleic acid by human gut bacteria: Different routes for biosynthesis of conjugated linoleic acid. J Bacteriol 189: 2566-2570.

Devkota S, Wang Y, Musch MW, et al. 2012. Dietary-fat-induced taurocholic acid promotes pathobiont expansion and colitis in Il10-/- mice. Nature 487: 104-8.

Duca F, Gérard P, Covasa M, Lepage P. Metabolic interplay between gut bacteria and their host. In: Delhanty PJD, van der Lely AJ, eds. How Gut and Brain Control Metabolism. Front Horm Res. Basel:
Karger, 2014, vol.42, pp.73-82. https://doi.org/10.1159/ 000358315

Faith JJ, Guruge JL, Charbonneau M, et al. 2013. The long-term stability of the human gut microbiota. Science 341 (6141):1237439. https://doi.org/10.1126/science.1237439.

Fleissner CK, Huebel N, Abd El-Bary MM, Loh G, Klaus S, Blaut M. Absence of intestinal microbiota does not protect mice from dietinduced obesity. 2010. Br J Nutr 104: 919-929. https://doi.org/ 10.1017/S0007114510001303.

Fu J, Bonder MJ, Cenit MC, et al. 2015. The Gut Microbiome Contributes to a Substantial Proportion of the Variation in Blood Lipids. Circ Res 117: 817-24. https://doi.org/10.1161/CIRCRE SAHA.115.306807.

Gérard P. 2014. Metabolism of Cholesterol and Bile Acids by the Gut Microbiota. Pathogens 3: 14-24.

Gérard P. 2016. Gut microbiota and obesity. Cell Mol Life Sci 73: 147-62. https://doi.org/10.1007/s00018-015-2061-5.

Gérard P. Gastrointestinal Tract: Microbial Metabolism of Steroids. In: Goldfine $\mathrm{H}$, ed. Health Consequences of Microbial Interactions with Hydrocarbons, Oils, and Lipids. Handbook of Hydrocarbon and Lipid Microbiology. Springer Nature Switzerland, 2020, pp. 389-399.

Gérard P, Bernalier-Donadille A. 2007. Les fonctions majeures du microbiote intestinal. Cah Nutr Diet 42: S28-S36.

Gérard P, Lepercq P, Leclerc M, Gavini F, Raibaud P, Juste C. 2007. Bacteroides sp. strain D8, the first cholesterol-reducing bacterium isolated from human feces. Appl Environ Microbiol 73: 5742-9.

Johnson EL, Heaver SL, Waters JL, et al. 2020. Sphingolipids produced by gut bacteria enter host metabolic pathways impacting ceramide levels. Nat Commun 11: 2471. https://doi.org/10.1038/ s41467-020-16274-w.

Just S, Mondot S, Ecker J, et al. 2018. The gut microbiota drives the impact of bile acids and fat source in diet on mouse metabolism. Microbiome 6: 134. https://doi.org/10.1186/s40168-018-0510-8.

Juste C. 2005. Acides gras alimentaires, flore intestinale et cancer. Bull Cancer 92: 708-721.

Kenny DJ, Plichta DR, Shungin D, et al. 2020. Cholesterol Metabolism by Uncultured Human Gut Bacteria Influences Host Cholesterol Level. Cell Host Microbe 28: 245-257. https://doi. org/10.1016/j.chom.2020.05.013.

Knight R, Callewaert C, Marotz C, et al. 2017. The Microbiome and Human Biology. Annu Rev Genomics Hum Genet 18: 65-86. https://doi.org/10.1146/annurev-genom-083115-022438.

Kriaa A, Bourgin M, Potiron A, et al. 2019. Microbial impact on cholesterol and bile acid metabolism: current status and future prospects. J Lipid Res 60: 323-332. https://doi.org/10.1194/jlr. R088989.

Kübeck R, Bonet-Ripoll C, Hoffmann C, et al. 2016. Dietary fat and gut microbiota interactions determine diet-induced obesity in mice. Mol Metab 5: 1162-1174. https://doi.org/10.1016/j. molmet.2016.10.001.

Le Chatelier E, Nielsen T, Qin J, et al. 2013. Richness of human gut microbiome correlates with metabolic markers. Nature 500: 5416. https://doi.org/10.1038/nature12506.

Le Roy T, Lécuyer E, Chassaing B, et al. 2019. The intestinal microbiota regulates host cholesterol homeostasis. BMC Biol 17: 94. https://doi.org/10.1186/s12915-019-0715-8.

Li J, Jia H, Cai X, et al. 2014. An integrated catalog of reference genes in the human gut microbiome. Nat Biotechnol 32: 834-841. https://doi.org/10.1038/nbt.2942.

Li H, Zhu Y, Zhao F, et al. 2017. Fish oil, lard and soybean oil differentially shape gut microbiota of middle-aged rats. Sci Rep 7: 826. 
Lloyd-Price J, Abu-Ali G, Huttenhower C. 2016. The healthy human microbiome. Genome Med 8: 51. https://doi.org/10.1186/ s13073-016-0307-y.

Martinez-Guryn K, Hubert N, Frazier K, et al. 2018. Small Intestine Microbiota Regulate Host Digestive and Absorptive Adaptive Responses to Dietary Lipids. Cell Host Microbe 23: 458-469. https://doi.org/10.1016/j.chom.2018.03.011.

Mokkala K, Houttu N, Cansev T, Laitinen K. 2020. Interactions of dietary fat with the gut microbiota: Evaluation of mechanisms and metabolic consequences. Clin Nutr 39: 994-1018. https://doi.org/ 10.1016/j.clnu.2019.05.003.

Müller VM, Zietek T, Rohm F, et al. 2016. Gut barrier impairment by high-fat diet in mice depends on housing conditions. Mol Nutr Food Res 60: 897-908. https://doi.org/10.1002/mnfr.201500775.

Muralidharan J, Galiè S, Hernández-Alonso P, Bulló M, SalasSalvadó J. 2019. Plant-Based Fat, Dietary Patterns Rich in Vegetable Fat and Gut Microbiota Modulation. Front Nutr 6: 157. https://doi.org/10.3389/fnut.2019.00157.

Palmer C, Bik EM, DiGiulio DB, Relman DA, Brown PO. 2007. Development of the human infant intestinal microbiota. PLoS Biol 5: e177.

Rabot S, Membrez M, Bruneau A, et al. 2010. Germ-free C57BL/6J mice are resistant to high-fat-diet-induced insulin resistance and have altered cholesterol metabolism. Faseb J 24: 4948-59.

Rabot S, Membrez M, Blancher F, et al. 2016. High fat diet drives obesity regardless the composition of gut microbiota in mice. Sci Rep 6: 32484.

Safari Z, Gérard P. 2019. The links between the gut microbiome and non-alcoholic fatty liver disease (NAFLD). Cell Mol Life Sci 76: 1541-1558. https://doi.org/10.1007/s00018-019-03011-w.

Safari Z, Monnoye M, Abuja PM, et al. 2019. Steatosis and gut microbiota dysbiosis induced by high-fat diet are reversed by 1week chow diet administration. Nutr Res 71: 72-88. https://doi. org/10.1016/j.nutres.2019.09.004.

Safari Z, Bruneau A, Monnoye M, et al. 2020. Murine Genetic Background Overcomes Gut Microbiota Changes to Explain
Metabolic Response to High-Fat Diet. Nutrients 12: 287. https:// doi.org/10.3390/nu12020287.

Thorasin T, Hoyles L, McCartney AL. 2015. Dynamics and diversity of the 'Atopobium cluster' in the human faecal microbiota, and phenotypic characterization of 'Atopobium cluster' isolates. Microbiology 161: 565-79. https://doi.org/10.1099/ mic.0.000016.

Velagapudi VR, Hezaveh R, Reigstad CS, et al. 2010. The gut microbiota modulates host energy and lipid metabolism in mice. $J$ Lipid Res 51: 1101-1112. https://doi.org/10.1194/jlr.M002774.

Vulevic J, McCartney AL, Gee JM, Johnson IT, Gibson GR. 2004. Microbial species involved in production of 1,2-sn-diacylglycerol and effects of phosphatidylcholine on human fecal microbiota. Appl Environ Microbiol 70: 5659-66. https://doi.org/10.1128/ AEM.70.9.5659-5666.2004.

Wan Y, Wang F, Yuan J, et al. 2019. Effects of dietary fat on gut microbiota and faecal metabolites, and their relationship with cardiometabolic risk factors: a 6-month randomised controlledfeeding trial. Gut 68: 1417-1429. https://doi.org/10.1136/gutjnl2018-317609.

Watson H, Mitra S, Croden FC, et al. 2018. A randomised trial of the effect of omega-3 polyunsaturated fatty acid supplements on the human intestinal microbiota. Gut 67: 1974-1983. https://doi.org/ 10.1136/gutjnl-2017-314968.

Wolters M, Ahrens J, Romaní-Pérez M, et al. 2019. Dietary fat, the gut microbiota, and metabolic health-A systematic review conducted within the MyNewGut project. Clin Nutr 38: 2504 2520. https://doi.org/10.1016/j.clnu.2018.12.024.

Zhang Y, Zhou S, Zhou Y, Yu L, Zhang L, Wang Y. 2018. Altered gut microbiome composition in children with refractory epilepsy after ketogenic diet. Epilepsy Res 145: 163-168. https://doi.org/ 10.1016/j.eplepsyres.2018.06.015.

Zheng CJ, Yoo JS, Lee TG, Cho HY, Kim YH, Kim WG. 2005. Fatty acid synthesis is a target for antibacterial activity of unsaturated fatty acids. FEBS Lett 579: 5157-62.

Cite this article as: Gérard P. 2020. The crosstalk between the gut microbiota and lipids. OCL 27: 70. 